\title{
Algunas notas sobre las primeras aplicaciones del hierro en la construcción de puentes en España
}

\author{
Alfonso LuJÁn DíAZ \\ Universidad Complutense de Madrid. Departamento de Historia del Arte III (Contemparáneo) \\ Centro de Estudios Históricos de Obras Públicas y Urbanismo (CEHOPU-CEDEX)
}

\begin{abstract}
RESUMEN
La incorporación española al panorama internacional de las construcciones en hierro se retrasó unas décadas. Las condiciones políticas, sociales, económicas o de recursos estaban muy alejadas de los presupuestos que llevaron a naciones como Inglaterra o Francia a convertirse en las abanderadas del nacimiento y desarrollo industrial europeo. Durante el período fernandino (1814-1833) se desatendieron propósitos dirigidos a promover un correcto desarrollo tecnológico de la nación para equipararla a otros países europeos. Tras el fracaso de algunas propuestas y la escasa repercusión de otras, será a partir de la reorganización del Cuerpo de Ingenieros de Caminos, y su representación estatal en la Dirección General, cuando en España se comience a valorar el hierro como material constructivo aplicado a grandes obras de ingeniería civil.
\end{abstract}

Palabras clave: Puentes de hierro; ingeniería civil; ingenieros s. XIX; Real Academia de Bellas Artes de San Fernando; materiales construcción.

\section{Some notes on the first applications of iron in the construction of bridges in Spain}

\begin{abstract}
The Spanish introduction to the international scene in iron construction was delayed a few decades. The political, social, economic or resources were far removed from the budgets that led to nations such as England or France to become the leaders of the birth and development of European industry. During the period Fernandino (1814-1833) were ignored purposes designed to promote proper technological development of the nation into line with other European countries. After the failure of some proposals and the limited impact of others, will be from the reorganization of the Corps of Engineers, and his state representation in the DG, when Spain begins to value the iron as a building material applied to the large civil engineering works.
\end{abstract}

Keywords: Iron bridges; civil engineering; engineers 19th century; Royal Academy of Fine Arts of San Fernando; building materials. 
La incorporación española al panorama internacional de las construcciones en hierro se retrasó unas décadas. Las condiciones políticas, sociales, económicas, demográficas o de recursos estaban muy alejadas de los presupuestos que llevaron a naciones como Inglaterra o Francia a convertirse en las abanderadas del nacimiento y desarrollo industrial europeo. Mientras en el Reino Unido ya se estaban modificando las estructuras y sistemas de producción tradicionales adaptándose al desarrollo que suponían los nuevos avances técnicos y las agilizadas relaciones mercantiles del nuevo librecambismo emergente allá por el último tercio del siglo XVIII, aquí, en España, aún se mantenía una producción de objetos muy tradicional basada en un sistema económico arancelario de control de las mercancías.

No obstante, y a pesar de esta demora, hemos de descubrirnos ante notables construcciones en hierro dadas en nuestro suelo que, si bien no han funcionado como referentes internacionales a nivel conceptual, estructural o tipológico - salvo raras excepciones - sí evidencian por su cantidad y calidad un patrimonio arquitectónico-ingenieril nada desdeñable.

Desde los años setenta del pasado siglo, tanto historiadores como arquitectos e ingenieros, llevan estudiando con interés el patrimonio industrial español, prestando especial atención a las novedosas tipologías que nacieron al amparo de la técnica a lo largo del siglo XIX, como los mercados, estaciones de ferrocarril, puentes de hierro, galerías comerciales, pabellones de exposición, etc ${ }^{1}$. De forma paralela, se está profundizando en estudios históricos dedicados a rescatar del olvido la importancia que tuvieron para la articulación del territorio nacional, durante ese siglo XIX, la creación y el desarrollo de construcciones de ingeniería civil, los nuevos sistemas de comunicación, las obras públicas o la arquitectura militar ${ }^{2}$. En este

1 Los estudios más relevantes son, a mi juicio: AGUILÓ ALONSO, Miguel, Forma y tipo en el arte de construir puentes, Madrid, Abada, 2008; ARENAS DE PABLO, Juan José, Caminos en el aire: los puentes, 2 vol., Madrid, Colegio de Ingenieros de Caminos, Canales y Puertos, 2002; CASTAÑER MUÑOZ, Esteban, La arquitectura del hierro en España: los mercados del siglo XIX, Madrid, Monografías de la Real Academia de Ingeniería, 2006; El Mundo de las Estaciones, catálogo de la exposición (Madrid, 1980), Madrid, Ministerio de Cultura, Dirección General de Bellas Artes y Archivos, 1981; FERNANDEZ TROYANO, Leonardo, Tierra sobre agua: visión histórica universal de los puentes, 2 vol., Madrid, Colegio de Ingenieros de Caminos, Canales y Puertos, 2004; HERNANDO CARRASCO, Javier, Arquitectura en España 1770-1900, Madrid, Cátedra, 2004; GARCIA MATEO, José Luis (dir.), Inventario de puentes ferroviarios de España, Madrid, Fundación de los Ferrocarriles Españoles, Doce Calles, Aranjuez, 2004; LOPEZ GARCIA, Mercedes, MZA Historia de sus estaciones, Madrid, Turner, 1986; NAVASCUES PALACIO, Pedro, Arquitectura y arquitectos madrileños del siglo XIX, Instituto de Estudios Madrileños, Madrid, 1973; PIZZA, Antonio, Arte y arquitectura moderna (1851-1933): del Crystal Palace de Joseph Paxton a la clausura de la Bauhaus, Barcelona, Ed. de la Universidad Politécnica de Cataluña, 1999; PUENTE, Moisés, Pabellones de exposición. 100 años, Barcelona, Gustavo Gilli, 2000; SAENZ RIDRUEJO, Fernando, Los ingenieros de caminos, Madrid, Colegio de Ingenieros de Caminos, Canales y Puertos, 1993; STRIKE, James (et al.), De la construcción a los proyectos: la influencia de las nuevas técnicas en el diseño arquitectónico, 1700-2000, Barcelona, Reverté, 2004.

2 Destaco: AGUILO ALONSO, Miguel, El paisaje construido: una aproximación a la idea de lugar, Madrid, Colegio de Ingenieros de Caminos, Canales y Puertos, 1999; ARTOLA, Miguel (et al.), Los ferrocarriles en España 1844-1943, 2 vol., Madrid, Servicio de Estudios del Banco de España, 1978; CAPEL, Horacio, De Palas a Minerva: la formación científica y la estructura institucional de los ingenieros militares en el 
sentido, mi aportación a este empeño por revalorizar nuestro patrimonio industrial pasa por referir en esta comunicación recientes datos extraídos de diferentes archivos y bibliotecas, de los cuales se desprende la posibilidad de adelantar algunos años la asimilación e incorporación del nuevo material de hierro en la ingeniería española.

\section{Referencias y primeras manifestaciones en España: la Comisión de Arquitec- tura de la Academia}

Hasta el levantamiento en Coalbrookdale del considerado primer puente de hierro, en 1779, se realizaron a lo largo de la historia moderna varios intentos de utilización del hierro como material constructivo en puentes fijos y colgantes, ya no sólo como elemento auxiliar sino estructural, así como se desarrollaron sistemas o modelos mecánicos que participaban de los principios aplicados en ellos. Uno de los autores que mejor ha recogido estos datos ha sido Jean Mesqui, quien hace referencia a un puente descrito por Filarete en su Tratado de Arquitectura (ca. 1461-1464), que parece evocar a un puente colgante; ciertos instrumentos y máquinas ideadas por Leonardo da Vinci en sus dibujos que sugieren dispositivos de rotación y elevación en puentes; menciona el famoso Ponte Strallato (principios s. XVII), de Faustus Verantius, inspirado en ejemplos de puentes chinos recogidos durante sus viajes; cita un Pont du fer estant au bout du pont de pierre, en Ramorantin (1556); o el puente metálico de tres arcos que se pensó levantar en Lyon, hacia 1719, sobre el río Saône - afluente del Ródano - y cuyo proyecto no se llevó a cabo ${ }^{3}$. Importante este último por tratarse del ejemplo que supuso el punto de partida sobre las reflexiones relativas a la potencial utilización de este nuevo material, pues hasta el siglo XVIII ningún autor trató el hierro como elemento estructural.

En España, las primeras noticias que tenemos datan de 1815. Ese año el maestro arquitecto Juan Bautista Belaunzarán presentó a la Academia de San Fernando un proyecto de puente sobre la ría de Bilbao, "vien sea de fierro, ó levadizo de madera", en sustitución de uno antiguo incendiado. Juan de Barcenilla, secretario de la Comisión de Arquitectura de la Academia de San Fernando, organismo encargado de valorar este tipo de expedientes, envió un oficio a su secretario general, Martín

\footnotetext{
s. XVIII, Barcelona, Serbal, Madrid, Consejo Superior de Investigaciones Científicas, 1988; CRESPO DELGADO, Daniel, El viaje del progreso: las obras públicas en el Viage de España de Antonio Ponz (1772-1794), Valencia, Generalitat Valenciana, Consellería d'Infraestructures i Transports, 2008; GONZALEZ YANCI, M ${ }^{\mathrm{a}}$ Pilar, Los accesos ferroviarios a Madrid. Su impacto en la geografia urbana de la ciudad, Madrid, Instituto de Estudios Madrileños, 1977; MADRAZO MADRAZO, Santos, El sistema de transportes en España 17501850, Madrid, Colegio de Ingenieros de Caminos, Canales y Puertos, Turner, 1984; SEGRE, Roberto, Historia de la arquitectura y del urbanismo. Países desarrollados siglos XIX y XX, Madrid, Instituto de Estudios de Administración Local, 1985.
}

MESQUI, Jean, Le pont en France avant le temps des ingénieurs, Paris, Picard, 1986. 
Fernández Navarrete, en el que suspende manifestar un dictamen en su Junta del 6 de julio de ese mismo año hasta enterarse de "otros antecedentes sobre este mismo asunto" . Puede entenderse como un síntoma de desconfianza hacia el nuevo material y la ausencia de obras de este tipo. Pero también debió de echarse de menos una presentación más extensa del proyecto, donde se incluyeran planos de los alzados, cortes y secciones, información de cálculos, costes, etc., como más adelante quedará reflejado en otras propuestas.

Otro diseño rechazado fue un proyecto de puente de hierro para el paso del Rey, sobre el Manzanares, que comunicaba el Palacio Real con la Casa de Campo. Esta idea fue presentada por José Díaz Alonso en 1816, pero en este caso la autoridad encargada de inspeccionar estas propuestas era el arquitecto real, Isidro González Velázquez ${ }^{5}$. En su informe, González Velázquez advirtió del peligro que supondría la elección del hierro para la construcción de puentes debido a los posibles robos a que pudiera incitar el material. Resulta una valoración un tanto débil para una personalidad de su cargo, pues no refiere otro tipo de factores de carácter técnico, constructivo o estético de más peso que sustenten su decisión. Sin embargo, no hay que olvidar que en numerosas empresas de este tipo en las que se hacía uso del hierro como material constructivo era muy habitual que los encargados en la dirección de obras concretasen con exactitud en la documentación los movimientos de las piezas, el lugar donde debían permanecer aseguradas hasta su uso, custodio de las mismas, etc. La práctica del robo de materiales era una tónica constante, de la que están repletos los archivos de causas relacionadas con ellas. Como hemos dicho, el puente de hierro de Díaz Alonso fue rechazado y hubo de esperarse trece años para que se realizara el paso del Palacio al Reservado de la Casa de Campo. Casualmente fue construido por Isidro González Velázquez, en piedra.

En 1820 se perdió una gran oportunidad para que en España se hubiese comenzado a trabajar sobre esta tipología de puentes de hierro. El por entonces embajador español en París, el Conde de Fernán Núñez, envió a la Academia un informe en el que incluía un Sistema de construcción de Puentes de hierro forjado, documento redactado en París por el arquitecto del Ministerio del Interior francés, Bernard Poyet, el 14 de octubre de $1819^{6}$. Pareciéndole bien a la junta, el 25 de febrero de 1820 Julián de Barcenilla lo reenvía al secretario general, Martín Fernández de Navarrete, para que lo examine. Sin embargo, apunta que "como las obras de arquitectura no pueden juzgarse por solo un escrito sin la demostracion en diseño, es de dictamen de informar á la Academia seria muy conveniente

4 Expedientes de obras públicas censuradas por la Comisión de Arquitectura, ARABASF (Archivo de la Real Academia de Bellas Artes de San Fernando), signatura 1-29-5-153.

5 Propuesta rechazada de puente de hierro de José Díaz Alonso, 1816, AGP (Archivo General de Palacio), Patrimonio, Casa de Campo, leg. 17.

6 Expedientes de obras públicas censuradas por la Comisión de Arquitectura, ARABASF, signaturas $1-29-5-216$ y $2-31-10$. 
que el Exmo Sr Embajador en Francia proporcionase un dibujo del Puente que se propone executar Mr. Payet en cuyo caso la Comision evaluará su informe mas extensivo, y con mayor conocimiento" [sic]. Del sistema constructivo de Bernard Poyet se extraen las siguientes ventajas frente a los empleados en piedra o madera: mayor solidez, economía en su construcción y conservación, posibilidad para lograr mayores luces, rapidez de ejecución, comodidad al construirse sin obstaculizar el paso, fácil de armar y desmontar "no quedando en su lugar mas que los Pilares, ventaja importantisima en tiempo de Guerra" [sic], entre otras. Rechazado por el Cuerpo de Puentes y Calzadas francés sus modelos son presentados a la Academia española.

Pese a la novedad de constituirse como un sistema de construcción, la Academia no ignoraba la existencia de obras relativas a la aplicación del hierro como material constructivo y sus posibilidades técnicas. Apenas seis días antes de dicho reenvío, Juan Pascual Colomer, bibliotecario de la Real Academia, escribe a Julián de Barcenilla "conforme al contenido de su oficio de 5 del corriente" exponiéndole la presencia en los anaqueles de la biblioteca ${ }^{7}$ de la obra de Robert Fulton Sobre el modo de perfeccionar los canales de navegación con varias observaciones acerca de la construccion de acueductos y puentes de madera y de yerro ${ }^{8}$, así como un Proyecto de un puente de un solo arco presentado al rey de Francia en 1783, de Vincent Montpetit ${ }^{9}$. El trabajo del ingeniero norteamericano, publicado en Londres en 1796, fue traducido al francés "le 29 Pluviose, l'an 6 de la République Françai$s e$ ", es decir, hacia el 17 de febrero de 1798, subrayándose en él todas las virtudes que atesoraba el nuevo sistema de construcción de puente de hierro en arco acompañado de varias planchas del mismo con detalles de las piezas que lo componían. Así mismo, desde 1805, la biblioteca de la Academia contaba con un tomo del Art de Bâtir ${ }^{10}$, de Jean-Baptiste Rondelet, "tratado teórico y práctico" donde se presentaban ciertas soluciones constructivas, como "el refuerzo de la mampostería con un sistema de anclajes de hierro como solución a los desplazamientos" y en cuyas sucesivas ediciones aparecerán grabados de puentes de hierro en arco - el de Coalbrookdale, Sunderland, Pont du Jardin du Roi, Pont des Arts - , y suspendidos, con un sesudo estudio despiezado de sus partes y métodos de construcción empleados. Sobre Montpetit, señalar que ya Jean-Rodolphe Perronet, fundador y director de

\footnotetext{
2-31-10.

Expedientes de obras públicas censuradas por la Comisión de Arquitectura, ARABASF, signatura

8 FULTON, Robert, A treatise on the improvement canal navigation, exhibiting the numerous advantages to be derived from small canals. And boats of two to five feet wide, containing from two to five tons burthen. Witn a description of the machinery for facilitating conveyance by water through the most mountainous countries, independent of locks and aqueducts: including observations on the great importance of water communications, with thoughts on, and desings for, aqueducts and bridges of iron and wood, London, I. and J. Taylor at the Architectural Library, High Holborn, 1796.

9 MONPETIT, Vincent de, Prospectus d'un pont de fer d'une seule arche, Paris, Chez l'Auteur, 1783.

${ }_{10}$ NAVARRETE MARTINEZ, Esperanza, "Adquisición de libros para la biblioteca de la Academia de San Fernando (1794-1844), en Academia, no 88, 1999, pp. 127-154.
} 
la École Nationale des Ponts et Chaussées, le había rechazado tres propuestas de puentes de hierro entre 1778 y $1783^{11}$.

El 26 de octubre de 1824 el arquitecto de la Real Academia de Bellas Artes de San Fernando, Antonio Goicoechea, expone a la Comisión de Arquitectura un diseño de Puente colgante de cadenas de fierro sobre el rio Cada-agua, en Burceña ${ }^{12}$. Lo interesante de esta obra - aparte de su novedad - es la implicación financiera de varios empresarios de las Ante-iglesias de Abando y Baracaldo en su ejecución, pues los ayuntamientos se mantuvieron al margen. Se debían aprovechar los arranques de un puente arruinado de piedra que por lo fangoso del terreno se había venido abajo en tres ocasiones desde el inicio de su construcción, cuatro años antes. La Comisión de Arquitectura en su junta del 3 de noviembre de ese mismo año reconoció el diseño del puente colgante y "deseando que esta clase de obras se propague en España á imitacion de los yá establecidos en Inglaterra, y pareciendo bien el pensamiento mereció su aprobacion" [sic].

El Ayuntamiento de Bilbao, animado por las ventajas que encerraban esta clase de obras, presentó el 4 de Mayo de 1825 una propuesta de Puente colgante de cadenas al secretario de la Comisión de Arquitectura de la Academia. Eligió para su ejecución a Antonio Goicoechea para realizarlo sobre el Nervión, a una legua escasa del anterior. También presentaron planos y proyectos algunos empresarios bilbaínos, teniéndose por conveniente adoptar el sistema de Goicoechea para su revisión, corrección y aprobación. Así ocurrió en la censura de la junta de 9 de junio de ese año, hallándose conforme el examen y aprobado el proyecto. Cuatro años después, el 13 de enero de 1829, el maestro arquitecto Antonio Goicoechea, residente en la Villa de Bermeo, fue declarado acreedor de la gracia de Arquitecto de Mérito "haciendo relación de sus meritos artisticos en las diferentes obras y comisiones que ha desempeñado" [sic].

Resulta interesante comprobar la vena industrial que siempre ha circulado por el territorio vasco. Tierra regada por caudalosos ríos que en su corto recorrido abandonan las montañas en busca del mar, parece un escenario propicio para el aprovechamiento de los recursos ofrecidos por la Naturaleza. La Revista Pintoresca de las Provincias Bascongadas, en un número publicado en 1846, aparece salpicada de artículos que refieren esta privilegiada condición natural donde se aúnan de manera casi mítica el genio fertilizador de los ríos que vivifican la industria fabril desarrollada a lo largo de su curso. En la misma revista, se reproduce un grabado del mencionado puente colgante de Burceña ${ }^{13}$ (fig. 1) - que luego aparecerá en el

11 JAMES, John G., "Some steps in the evolution of early iron arched bridge designs", en Transactions of the Newcomen Society, no 59, 1987-1988, pp. 153-187. Apud AGUILÓ ALONSO, Miguel (2008), op. cit., p. 322.

12 Expedientes de obras públicas censuradas por la Comisión de Arquitectura, ARABASF, signatura 2-31-10

13 Existe cierta confusión, en diferentes artículos y libros, relativas a la fecha de realización y posterior destrucción durante las guerras carlistas de este puente, siendo difícil de esclarecer a cual de los dos puentes 
Diccionario de Pascual Madoz al tratar la provincia de Vizcaya - "porque no puede ménos de llamar la atencion el que haya construido [D. Antonio de Goicoechea] en España el primer puente colgante en un apartado lugar como Baracaldo" [sic]. No es ocasión para detenerse en este detalle, pero sin duda debe señalarse este guiño "pintoresco" por parte de la dirección de la revista de intercalar una lámina

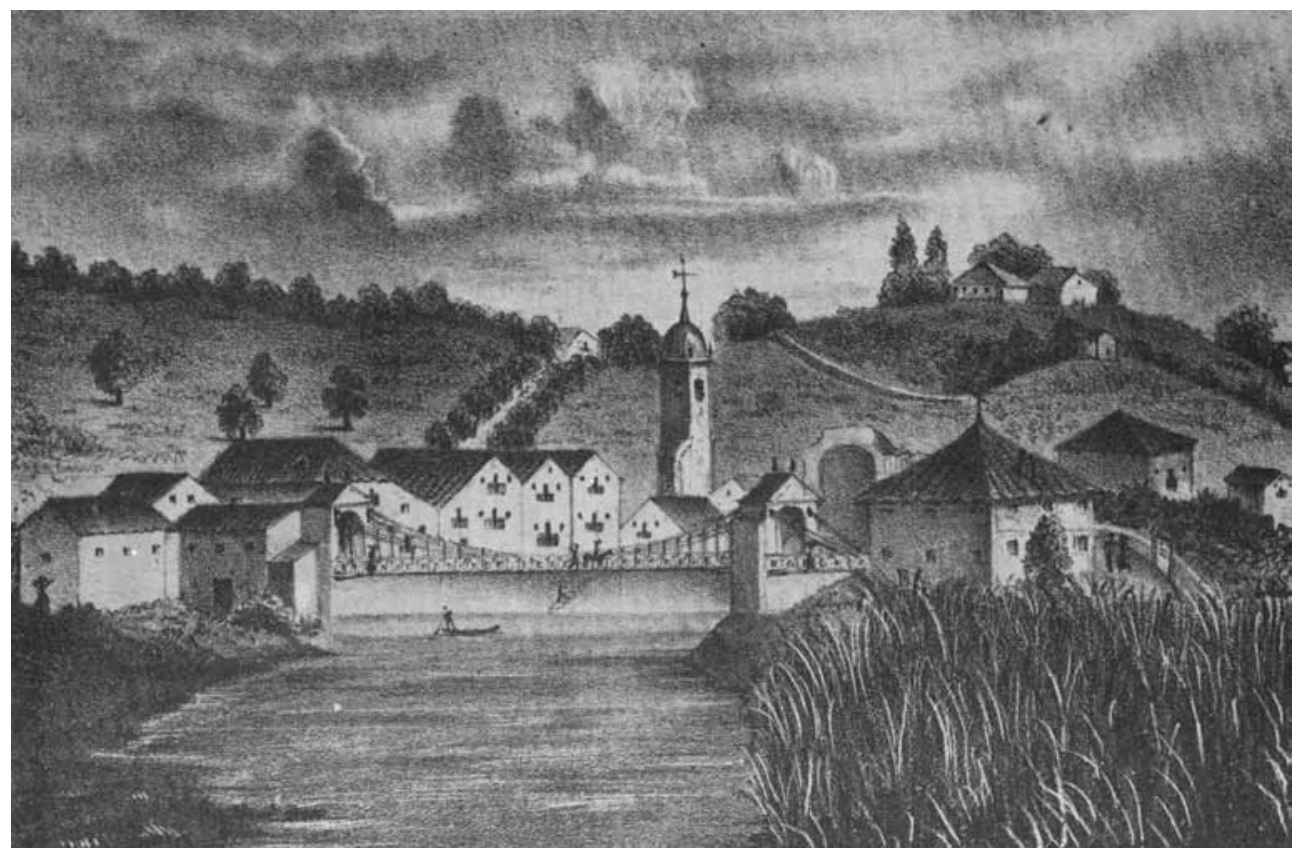

Fig. 1. Antonio de Goicoechea, Puente colgante de Burceña, Baracaldo (Vizcaya), 1824. Grabado [tomado de Revista Pintoresca de las Provincias Vascongadas, Bilbao, 1846, p. 349].

de un Puente colgante de hierro entre otras que representan los monumentos más notables de la antigüedad, pues como sostiene su autor "parécenos que formará un bello contraste" 14 .

Años después, el 22 de noviembre de 1830, el administrador del Real Heredamiento de Aranjuez, manifiesta la imperiosa necesidad de reestablecer la comunicación por el titulado Puente de Barcas que había quedado en estado ruinoso tras

aludidos más arriba se refiere. Por señalar algunas: IBARRETA, D. A., "Reconstrucción del Puente de Burceña", en Anales de la Construcción y de la Industria, nºs. 1, 12, 13, año V, 1880, pp. 1-4, 177-180, 193-199; y n ${ }^{\circ}$ s. 6 y 7, año VI, pp. 81-85, 97-100, respectivamente. FULLAONDO, Juan Daniel, La arquitectura y los arquitectos de la región y el entorno de Bilbao, vol. 2, Madrid-Barcelona, Alfaguara, 1969, p. 274; ALDANA, José Patricio, Puentes de Bilbao Bilbao, Área de Obras y Servicios del Ayuntamiento de Bilbao, 2000. Una vez revisada la documentación no cabe duda que este grabado representa el llamado Puente colgante de cadenas de fierro sobre el rio Cada-agua, de 1824.

14 Revista Pintoresca de las Provincias Vascongadas, Bilbao, 1846, pp. 349-350. 
"la estraordinaria avenida del Tajo en los tres primeros dias del presente mes" [sic]. $\mathrm{El}$ arquitecto mayor de $\mathrm{S}$. M., Isidro González Velázquez, tras varias inspecciones de la zona damnificada, presentó para el lugar unos diseños de un puente de cantería de un solo arco de 100 pies de diámetro con la intención de evitar disponer tajamares que presentasen resistencia al paso de las aguas. Para este fin también presentó el ingeniero de caminos Pedro Miranda un proyecto de un Puente suspendido con cadenas, que finalmente se llevó a cabo. Los motivos decisivos para que triunfase este último proyecto quedan reflejados por la magnífica propuesta de Miranda y sus posibilidades futuras, a pesar del alto presupuesto que estimó en 543,866 reales vellón ${ }^{15}$ :

“[D. Pedro Miranda] ... manifiesta las ventajas que ofrece á los Puentes de piedra, por su hermosura, duracion y economico en su coste; y añade que los Ingenieros y obreros ingleses empleados en el nuevo molino de aranjuez conocidos por su destreza y habilidad en el trabajo del hierro forjado y fundicion del colado, cooperarian á la perfecta egecucion de la parte de hierro, pudiendo hacerse todo en Aranjuez estableciendo los hornos y talleres correspondientes para los mencionados trabajos, y luego de concluidos estos quedarian aquellos talleres bajo la direccion de los mencionados ingleses en estado de surtir al publico de una multitud de obgetos y utensilios de hierro colado baratos, y de construir toda especie de maquinas, con lo cual se conseguirian dos obgetos que son construir una obra perfecta y acabada y plantear un nuevo ramo de industria en beneficio del pais" [sic].

Una nota lacrada del Despacho de la Mayordomia Mayor, con fecha de 22 de enero de 1831, declara inflexible: "Hagase el puente colgado". [sic]. Y así, se principiaron las obras. Para el 1 de agosto del año siguiente ya estaba muy avanzada la obra encontrándose dispuestas y "armadas las cadenas principales de suspension, en la misma disposicion que han de ocupar en el Puente" [sic]. Los hermanos Zavala, con ferrería en Tolosa, habiendo recibido las barras de hierro colado de Inglaterra, se encargaban de forjarlo para su posterior envío a Aranjuez, su montaje y pruebas de carga. En total el puente constaba de 2.625 arrobas y 9 libras de hierro forjado, distribuidos en " 12 segmentos de eslabon para las curvas de las pilastras, 90 eslabones de las 6 cadenas de suspension, 24 id. de los 6 tirantes de retencion, 80 colgantes verticales, 31 barras horizontales de apoyo $\mathrm{p}^{\mathrm{a}}$ el pavimento, 28 eslabones de respeto, 12 anclas ó pasadores $\mathrm{p}^{\mathrm{a}}$ los tirantes, 4 planchas $\mathrm{p}^{\mathrm{a}}$ la máquina de ensayar, 9 tercios con piezas pequeñas, como cajas, cabillas, llaves \&, 5 id. con pasadores para el pavimento" [sic]. El asentista y encargado a cuenta del herraje de Tolosa para el puente colgante de Aranjuez era Alejandro López Mollinedo, del comercio de Madrid. No obstante, se demorará la recepción de los materiales hasta los días 26 y 27 de agosto de 1833.

15 Se puede seguir la gestación y el proceso de evolución de las obras de este puente en: Puente colgado de Aranjuez 1830-1834, AGP, Administraciones Patrimoniales, Caja 14.318; algunos datos complementarios sobre el ingeniero en: Pedro Miranda. Expediente personal (serie), AGP, Personal, Caja 686, Exp. 40 
Hasta aquí, de lo expuesto se desprenden dos conclusiones: por un lado, la ausencia de una estructura estatal capaz de gestionar los expedientes de obras de esta índole mediante la aplicación de unos procedimientos administrativos prácticos; y por otro que, a falta de este referente público, el organismo competente responsable de estas materias seguía siendo la Comisión de Arquitectura de la Academia de San Fernando, salvo el caso del puente de Aranjuez al tratarse de una obra inserta en terrenos de patrimonio real. Por tanto, la escasa atención prestada al fomento y desarrollo de las obras públicas desde la administración central a lo largo del período fernandino, así como los daños materiales causados por las constantes guerras libradas en nuestro territorio pusieron de manifiesto la necesidad de una articulación territorial en base a proyectos de ingeniería civil que comenzaron a tomar cuerpo tímidamente a finales de la década de los años 30 .

\section{Implicación estatal: la Dirección General de Caminos}

A partir de 1833, ya durante el período de Regencia de María Cristina que sucedió a la muerte de Fernando VII, una serie de instrucciones y reales decretos dieron como resultado la reorganización de la Escuela de Caminos, la creación de la Dirección General de Caminos - ahora separada de la de Correos - compuesta por facultativos dependientes de la administración pública, o el reglamento de organización del propio Cuerpo de Ingenieros de Caminos. Desde ahora, esta Dirección General de Caminos será la responsable de examinar y tramitar toda obra pública planteada.

Así, el punto de inflexión que supuso la confirmación plena del empleo del hierro en los puentes españoles fue la negociación, desde la Dirección General de Caminos, para llevar a cabo la construcción de varios puentes colgantes ofrecidos por el ingeniero civil y empresario francés Jules Seguin. Se trataba de uno de los hermanos y colaborador del prestigioso ingeniero Marc Seguin, junto con el que estudió la tracción de los cables metálicos desde 1822 y su consiguiente aplicación en puentes suspendidos en sustitución de las cadenas, pues comenta G.H. Dufour que "tras pruebas a pequeña escala se convencieron de poder realizarlos sobre los mismos principios de los puentes colgantes de cadenas de Inglaterra y América"16. Los ingenieros franceses tenían conocimiento de las experiencias llevadas a cabo en Inglaterra en puentes colgantes de cadenas gracias a sus visitas a este país. Hacia 1820 ya pudieron ver concluido el Union Bridge, diseñado por el oficial de marina Samuel Brown sobre el río Tweed, en Escocia, primera manifestación de puente suspendido con cadenas de

16 DUFOUR, Guillaume Henri, Description du pont suspendu en fil de fer, construit a Genève, Genève, Chez J.J. Paschoud et Paris, Même Maison du Commerce, 1824. No obstante, la utilización de un sistema de "fils de fer" para sustentar el palastro ya estaba siendo empleado en Francia por el banquero, político, industrial, hombre de ciencia y filántropo Benjamin Delessert para unir una de sus fábricas con su residencia mediante un puente colgante de 52m. de longitud en Passy, junto al Sena: Notice sur le pont suspendu en chaîne et fil de fer, de Passy, près Paris, par Benjamin Delessert, Paris, 1824. 
hierro. También pudieron tener acceso al Treatise on bridge architecture (1811), del ingeniero Thomas Pope, célebre descripción de los primeros puentes colgantes que James Finley exportó a los EE.UU. ${ }^{17}$ Los ensayos de los hermanos Seguin avalaban el inicio de una larga serie de realizaciones que iban a tener por principal objetivo salvar el Ródano, exportándose esta innovadora tipología al resto de Francia y Europa. Se calcula que hacia 1841 habían levantado más de 75 puentes suspendidos.

Desde 1837 se vino dando vueltas a la idea de contratar la propuesta del ingeniero Jules Seguin. En ella se encargaba de establecer "á sus costas, riesgos y peligros, Puentes Colgantes en los puntos y sobre los Rios siguientes" [sic]: sobre el Tajo en Fuentidueña; sobre el Jarama en Arganda; sobre el Pas en Carandia, a poca distancia de Santander; sobre el Júcar en Cullera; sobre el Gállego cerca de Zaragoza; el de San Alejandro en el Puerto de Santa María, y otro sobre el río San Pedro; sobre el Guadalquivir en Sevilla; otro sobre el mismo río en Menjíbar; y restablecer la comunicación del Puente de Almaraz, sobre el Tajo, arruinado tras la I Guerra Carlista. En total nueve puentes colgantes y un trabajo de rehabilitación. La importancia que suponía llevar a buen fin esta empresa quedaba patente desde bien temprano en el ánimo de la Dirección de Caminos, ya que el 24 de mayo de ese año manifestaba "la importancia de promover las obras publicas con el fin de evitar los males que el estado de nuestras comunicaciones ocasiona, y producir ventajas considerables al País" [sic]. No obstante, tan sólo se levantaron cuatro de ellos, ya ejecutados bajo la Sociedad Española de los Cuatro Puentes Colgantes, filial española de la marca francesa de los Hermanos Seguin.

Durante más de tres años se suceden continuos intercambios de impresiones y modificaciones en los términos de la contrata entre la Dirección general de Caminos, Canales y Puertos, el entonces apoderado general en España del ingeniero francés, Vicente Luis Cusin, y el propio Jules Seguin. Finalmente, en Madrid a 21 de diciembre de 1840, firman la escritura de contrata de concesión de dichos puentes Francisco Javier Barra, subinspector y encargado de la Dirección General de Caminos, de una parte, y de la otra Manuel de Marliani, cónsul general de S.M. en París, representando al ingeniero Seguin ${ }^{18}$.

Del pliego de condiciones de la contrata se extraen ciertas cuestiones que revelan de manera directa la fragilidad estructural que aún existía en la administración a la hora de acometer empresas de gran envergadura. En primer lugar, transcurren casi cuatro años desde la oferta de Seguin hasta la firma del contrato, con reiteradas rectificaciones y cambios en sus términos que evidencian una falta de autoridad competente por parte de la Dirección de Caminos ante la ausencia de un modelo

17 PICON, Antoine, "Navier and the introduction of suspension bridges in France", en SUNDERLAND, R. J. M. (ed.), Structural iron, 1750-1850, Studies in the History of Civil Engineering, vol. 9, Aldershot, Ashgate Variorum, 1997, pp. 211-224.

${ }_{18}$ La siguiente referencia guarda todo lo relacionado con esta contrata: Expediente General (proyectos) de los puentes colgantes. 1837. AGA (Archivo General de la Administración), Sign. (04) 24/05814. 
de contratas al uso. En segundo lugar, esta sensación de hieratismo institucional se veía incrementada por el constante obstáculo que suponía el coste total de las obras y su manera de financiación, quizás uno de los factores al que se le dio mayor relevancia durante este proceso. De hecho, sobre el coste de los puentes se planteaba una inseguridad fundamental motivada por su propia novedad, pues "si los puentes se construyesen de piedra ó de madera podrian hacerse calculos bastante aproximados para esta clase de contratas alzadamente: pero se han construido tan pocos colgados en España y estos bajo diferente sistema del que se propone emplear Mr. Seguin, que es extremadamente dificil hacer un calculo aproximado fundado" [sic]. Por ello, la única referencia con la que se contaba para tasar el precio de estas nuevas construcciones era la desprendida de otros puentes realizados en piedra con unas dimensiones y características de emplazamiento similares. La financiación de los mismos habría de regularse con criterios semejantes, en este caso mediante un cálculo que tendría como base orientativa el producto extraído de los pasos de barcas establecidos anteriormente.

Sin embargo, otros artículos del expediente manifiestan una plena consciencia institucional a la altura de las circunstancias. En ellos se fijan condiciones elementales a obedecer, como la sujección del empresario Seguin a "inspección inmediata del Ingeniero ó Ingenieros que nombre el Director general" [sic], la imposibilidad de "subarrendar ó traspasar la obligacion contenida en esta contrata á ninguna persona" [sic] debiendo construir los puentes bajo su responsabilidad y nombre, que acompañe al proyecto una relación "que acerca de la composicion de las cadenas ó cables de suspension manifieste los datos y calculos necesarios para asegurarse que su macsima tension en la carga de prueba está en los limites indicados" [sic], la obligación de mantener "en el mejor estado y á su costa" [sic] los puentes durante el tiempo de la concesión, o permitir durante el transcurso de las obras "espedito el paso sea por el mismo puente, sea por barca ó por otro medio con la debida comodidad en el concepto de que si por descuido ó culpa suya sufriere el servicio publico la Direccion general podra tomar las medidas oportunas para poner corriente la comunicación rebajando de las anualidades que corresponden al Empresario los gastos que para ello hiciere la Direccion" [sic].

Estos términos de la contrata muestran algunos compromisos a los que quedaba sujeto el empresario e ingeniero, si bien por otro lado ofrecía ciertas facilidades para el correcto desarrollo de la obra. La Dirección General de Caminos se comprometía a entregar a Jules Seguin los terrenos que fueran a ocupar los estribos y demás espacios necesarios para el establecimiento del puente, siendo "de cuenta de este [Seguin] las inmediaciones de daños y perjuicios de los ocupados eventualmente para talleres ó materiales" [sic]. Se le concedían "todas las franquicias de obras publicas, para los comestibles de los operarios empleados en las obras, la abertura de Canteras, para cortar leña y hacer uso de los pastos comunales" [sic]. Aunque quizás una de las claúsulas más importantes era la total libertad de "introducir en España franco de todo derecho y alcabalas los hierros, cobre, hierro colado 
y alambres necesarios á la construccion de los puentes" [sic]. Así mismo, Seguin quedaba exento de cualquier "especie de contribuciones generales, provinciales y municipales, ni de patente, subsidio ó cualquiera otra ecsistente ó que se establezca en lo sucesivo" [sic] durante el tiempo de concesión de la contrata, que en este caso iban de 40 a 50 años, dependiendo de los rendimientos de los productos extraídos de los pontazgos.

\section{Hacia una valoración estética del material}

El 1 de diciembre de 1844, el ingeniero de Caminos, Canales y Puertos, y director de las obras de la Carretera de las Cabrillas para establecer una moderna comunicación entre la capital y Valencia, Lucio del Valle, presentó a la Academia de San Fernando una Memoria sobre la situación, disposición y construcción de los Puentes para su nombramiento como Académico de Mérito de la misma ${ }^{19}$. A lo largo de sus líneas hace un breve repaso histórico y técnico de los diferentes tipos de puentes adoptados en construcción: de barcas, de madera, levadizos o giratorios, de fábrica, de hierro fijos y colgantes. En lo relativo a estos dos últimos modelos, y sirviéndose de ejemplos recientes - como el Pont du Carrousel, de A. R. Polonceau - alaba las magníficas propiedades técnicas y economía de tiempo y coste de aquéllos en los que el hierro se emplea como material estructural portante, algo ya por entonces muy asumido en la literatura especializada. Sin embargo, cabe destacar el prudente juicio que declara sobre una supuesta valoración estética de puentes de esta tipología. Ante esta cuestión, manifiesta con cautela la necesaria aclimatación de estos sistemas constructivos para su correcta valoración, ya que "en mi concepto, aun no es tiempo de fijar definitivamente esta cuestion: es preciso para ello, que pasen bastantes años para que las observaciones que se hagan con esactitud y sin espiritu de partido, puedan tenerse en cuenta" [sic]. La principal duda que asaltaba a la hora de opinar sobre este tipo de trabajos era la falta de solidez que podían evidenciar estas estructuras frente a la alcanzada por la piedra. En el fondo, sobrevolaba el debate fundamental que habría de cruzar, a lo largo de todo el siglo XIX, los núcleos académicos y técnicos europeos, con sus consiguientes discusiones y publicaciones en revistas especializadas: la disociación y convivencia entre el tradicional arquitecto/artista creador de belleza y el moderno ingeniero/ técnico como ser útil ${ }^{20}$. En el caso de los puentes colgantes, del Valle se atreve a indicar su preferencia por los de alambre - introducidos por el antedicho Marc Seguin - frente a los de cadenas, si bien esta defensa pasaba por cuestiones puramente técnicas - ahorro de material, mayor consistencia, facilidad en el manejo y

19 VALLE, Lucio del, Memoria sobre la situación, disposición y construcción de los puentes: Valencia, 1844, Madrid, Fundación Esteyco, 1994.

${ }^{20}$ CORELLA SUÁREZ, Pilar, Puentes madrileños de la etapa isabelina, Madrid, Instituto de Estudios Madrileños del C.S.I.C, 1993. 
colocación de los cables, deseables a la hora de su conservación, etc. - alejadas de cualquier estimación estética.

En los años 40 del siglo XIX, aún se destacaba por encima del valor artístico de estas piezas su interés práctico, utilitario y económico; apenas se intuía una disposición intelectual para enjuiciarlas desde el punto de vista del arte. Únicamente han aparecido, casi de manera trivial, algunos términos descriptivos que podemos considerar se aproximan a categorías elevadas: bello, curioso, hermoso. Con la aparición de las primeras publicaciones europeas dedicadas por completo a la reflexión en el terreno de la técnica y la ingeniería, y la extensión a todos los campos de la construcción del empleo del hierro a gran escala provocadas por el auge de las exposiciones universales quedará planteado de manera sólida ese debate de las competencias profesionales entre arquitectos e ingenieros y la consiguiente estimación del material como viable elemento de renovación formal en el panorama constructivo. 